\title{
Comportamiento social del mono capuchino común Cebus olivaceus (Primates: Cebidae) en tres exhibiciones zoológicas de Caracas, Venezuela
}

\author{
Marie Charlotte López ${ }^{1} \&$ Zaida Tárano ${ }^{2}$ \\ 1. Universidad Central de Venezuela, Facultad de Ciencias, Escuela de Biología, Caracas, Venezuela; \\ charliecs@yahoo.com \\ 2. Universidad Central de Venezuela, Instituto de Biología Experimental, Apartado Postal 47106, Caracas, Venezuela; \\ zaida.tarano@ciens.ucv.ve
}

Recibido 11-VII-2006. Corregido 30-VI-2008. Aceptado 31-VII-2008.

\begin{abstract}
Social behavior of the Wedge-capped Capuchin Monkey Cebus olivaceus (Primates: Cebidae) in three zoological exhibits of Caracas, Venezuela. Captivity represents an extreme situation for primates, especially for those with large home ranges, and its effect on their behavior might be considerable. The Wedgecapped Capuchin Monkey Cebus olivaceus is the most common primate in Venezuelan zoos. To estimate the effect of confinement on C. olivaceus behavior, we analyzed the social behavior of three groups that differed in captivity conditions, in zoological exhibits in Caracas (Caricuao, Parque del Este, El Pinar). Caricuao's group moved freely over a non-fenced area of 15 ha, Parque del Este's and El Pinar's groups lived in relatively small outdoor enclosures. Social behaviors were described using focal-animal sampling, group scans and ad libitum sampling. The frequency, duration and time devoted to each behavior (per focal period per individual) were estimated. Relative dominance between pairs of individuals was established as well as affiliative associations. The repertory of social behaviors was similar between groups and to which has been observed in nature, but the duration and frequency of affiliative and agonistic interactions differed between groups. Affiliative behaviors were less frequent but longer in Caricuao than in the other two groups, while agonistic behaviors were more frequent in El Pinar and Parque del Este. Differences between groups are explained by variation in captivity conditions. We suggest that confinement generates social tension and favors agonism, while affiliative encounters help reduce this tension. On the other hand, differences in agonism between captive and natural groups may result form prolonged association, restrictions to keep optimal spacing or leave the group. All groups had some social structure (e.g., dominance ranks, association and repulsion between individuals) but the social dynamic was partly disrupted. Dominance ranks were not clear throughout the group, the top male was not dominant over the top female, dominant individuals did not interact affiliatively more than other individuals, and females did not affiliate frequently with other females. All of these observations are contrary to which has been observed in nature. As a whole, C. olivaceus tolerates captivity well because its behavioral repertoire is similar to that in natural conditions, and abnormal or undesirable behaviors (e.g., self-mutilation, stereotyped actions), were not observed. Nonetheless, there is an effect of captivity, reflected in a disruption of the social dynamic. Rev. Biol. Trop. 56 (3): 1503-1520. Epub 2008 September 30.
\end{abstract}

Key words: sociality, agonism, affiliation, dominance, captivity, social structure, tropical primate, Cebus olivaceus, Wedge-capped Capuchin monkey.

Los primates son animales de exhibición frecuente en los zoológicos porque muestran conductas y estructuras sociales similares a las de los humanos. Las especies comúnmente encontradas en zoológicos de Europa y Norteamérica son los chimpancés (Pan troglodytes), los gorilas (Gorilla sp.), los orangutanes
(Pongo sp.), los babuinos o mandriles (Papio spp.) y varias especies de macacos (Macaca spp.). La mayoría de los zoológicos modernos exhiben a los grandes simios (gorilas y orangutanes) en encierros amplios, pero muchos todavía mantienen a los chimpancés y los monos en jaulas pequeñas (de Waal 1989). 
El confinamiento crea condiciones nuevas para los primates, especialmente para aquellos que en condiciones naturales tienen áreas de vivienda grandes. Las conductas típicamente asociadas con actividades rutinarias como buscar alimento, seleccionar pareja o dispersarse no pueden expresarse o lo hacen sólo parcialmente (Goldblatt 1993). Además, los miembros del grupo y la dieta son impuestos. Los grupos presentes en los zoológicos se forman por la introducción de individuos provenientes de circos, otras exhibiciones zoológicas, hogares humanos o de ambientes naturales, y su composición puede variar en cualquier momento. Numerosos estudios con animales en cautiverio mencionan la aparición de conductas atípicas como moverse continuamente de un lado a otro, interacciones agonísticas frecuentes e intensas y auto mutilación (Mallapur y Choudhury 2003); además, estas conductas son más probables en animales que provienen de hogares humanos o nacidos en zoológicos que en los criados en condiciones naturales (Mallapur et. al. 2005).

La conducta social de los primates ha sido analizada ampliamente debido a su importancia en la estructura y mantenimiento de los grupos, en el desarrollo de ciertas habilidades y conductas individuales (Visalberghi y Addessi 2000) y a que es muy sensible a los cambios en el ambiente externo e interno (Hosey 2005). Los comportamientos sociales observados en tropas de primates han sido divididos en varias categorías generales (Richards 1974): afiliativos, agonísticos, sexuales, juego social e interacciones materno filiales. En la estructura y el mantenimiento de los grupos son especialmente importantes las conductas afiliativas y las agonísticas. Las conductas afiliativas no sexuales o reproductivas, median o promueven nexos entre los individuos, mientras que las agonísticas son interacciones competitivas o de conflicto de intereses, ya sean de sumisión, agresión directa o mediante despliegues ritualizados (Grier y Burk 1992), asociadas al acceso a recursos. En primates sociales, el agonismo también conduce al establecimiento de órdenes de rango y jerarquías que mantienen la cohesión del grupo aún cuando existan rivalidades (Richards 1974).

El mono capuchino común Cebus olivaceus Schomburgk, 1848 (Cebidae, Cebinae) es el primate más común en los zoológicos venezolanos. Algunos aspectos de la historia natural del género han sido descritos en cautiverio y en ambiente natural (Robinson 1981, Fragaszy et al. 1990, Welker et al. 1987, 1990, Fedigan 1993, Kinzey 1997, Manson y Perry 2000), pero los trabajos con C. olivaceus son relativamente escasos. En Venezuela, C. olivaceus ocurre en bosques de galería y de arbustos, y forma grupos constituidos por varios machos y hembras, con un macho y una hembra dominantes en un grupo de 8 a 36 individuos, dependiendo del hábitat (Robinson 1981, Kinzey 1997). En cuanto a la composición del grupo, el número de hembras adultas es generalmente mayor o al menos igual al número de machos adultos y los jóvenes y adultos están aproximadamente en igual proporción (Freese y Oppenheimer 1981). La jerarquía está basada en la edad, en hembras como en machos. La jerarquía de las hembras es matrilineal y la hembra de más rango domina a todos los miembros del grupo excepto al macho alfa. La mayoría de las interacciones agonísticas involucran a los individuos de alto rango; los machos de rangos inferiores evitan el contacto o la aproximación a machos de mayor rango (Izawa 1980). El acicalamiento es la actividad más frecuente durante los períodos de descanso y los animales dominantes son los que más acicalamiento reciben. El juego social entre infantes, juveniles y subadultos es común (Rowe 1996). En cuanto a la dieta, C. olivaceus consume invertebrados y frutas, aunque también puede consumir vertebrados y huevos de aves (Freese y Oppenheimer 1981). El tamaño del área de vivienda depende de la distribución de las frutas (Kinzey 1997).

A pesar de su frecuencia en los zoológicos venezolanos, no existen publicaciones del comportamiento del mono capuchino en estas condiciones (Levy 1984). Aunque los reportes de alteraciones del comportamiento social en primates en cautiverio son relativamente frecuentes, la sensibilidad de los individuos 
a esta situación artificial varía ampliamente entre especies y entre exhibiciones (Mallapur y Choudhury 2003). Con el fin de analizar el efecto del cautiverio en el comportamiento social del mono capuchino común, C. olivaceus, se realizaron observaciones en tres zoológicos de Caracas que diferían en la calidad del encierro. Específicamente se estableció el repertorio de conductas sociales y se describió cualitativa y cuantitativamente. También se analizó la dinámica social de los grupos a través de las relaciones agonísticas y afiliativas entre los individuos. Los resultados se discuten considerando las diferencias en las condiciones de cautiverio entre los zoológicos y en comparación con lo descrito en condiciones naturales.

\section{MATERIALES Y MÉTODOS}

Sitio de estudio: el estudio se realizó en tres parques que mantienen exhibiciones zoológicas en la ciudad de Caracas, Venezuela: Parque Zoológico Caricuao $\left(10^{\circ} 25^{\prime} 50^{\prime \prime} \mathrm{N}, 6^{\circ} 58^{\prime} 03^{\prime \prime}\right.$ W), Parque del Este Generalísismo Francisco de Miranda $\left(10^{\circ} 29^{\prime} 34^{\prime \prime} \mathrm{N}, 66^{\circ} 50^{\prime} 19^{\prime \prime}\right.$ W) y Parque

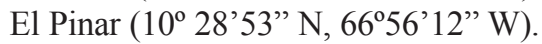

El Parque Zoológico Caricuao se encuentra en el sudoeste de la ciudad y cubre 630 hectáreas de las cuales 36 son áreas verdes de acceso al público. El grupo observado se desplazaba libremente en una porción de las áreas de acceso al público y en la ladera de las colinas restringidas a los visitantes (0.06 ind/ha).

El Parque del Este se encuentra en el este de Caracas y se extiende sobre 82 hectáreas, de las cuales 75 son de acceso al público. Los monos se encontraban en un área cercada de aproximadamente $450 \mathrm{~m}^{2}$ que contenía dos islas artificiales, unidas entre sí por un puente de madera, y rodeadas por agua. El área total de estas islas era aproximadamente $246 \mathrm{~m}^{2}$ $\left(0.045 \mathrm{ind} / \mathrm{m}^{2}\right)$. En las islas había árboles, un comedero, casetas de madera, palos verticales y cuerdas. Los individuos eran encerrados en unas jaulas subterráneas en la isla más grande a las 1700 h, y liberados al día siguiente a las $0730 \mathrm{~h}$.
El Parque Zoológico El Pinar se encuentra en el sudoeste de Caracas y su extensión es de 7 ha aproximadamente. Los monos se encontraban en una fosa de aproximadamente 200 $\mathrm{m}^{2}$ que contenía una isla rodeada por agua de profundidad variable $\left(0.053 \mathrm{ind} / \mathrm{m}^{2}\right)$. La isla era plana, con palos, troncos, cuerdas y dos columpios. Todos los grupos eran alimentados con frutas, zanahoria y maíz, entre las 0730 y las $0800 \mathrm{~h}$.

Objetos de estudio: cada individuo fue identificado a partir de marcas individuales naturales como el patrón de coloración y la distribución del pelaje, cicatrices, contextura u otros, fácilmente observables a distancia. Se utilizó un código de tres a cinco letras, las dos primeras representan el sexo $(\mathrm{M}, \mathrm{H})$ y la edad del individuo (A: adulto; J: juvenil; Cr: cría o infante; ?: no determinado), las restantes la identidad individual. El grupo de Caricuao estaba formado por 11 individuos: 6 machos ( 2 adultos) y 5 hembras (3 adultas): MA-MR, MA-MC, M?-BB, M?-FL, MJ-TA, MJ-CO, HA-MA, HA-NEG, HA-CB, HJ-TI, HJ-CH. Durante el periodo de observación nacieron tres crías: Cr-MA (de HA-MA), Cr-NE (de HA-NEG) y Cr-CB (de HA-CB).

El grupo del Parque del Este estaba formado por 12 individuos: 8 hembras (4 adultas) y 3 machos (un adulto) y un infante: HA-NE, HA-MCR, HA-B, HA-PN, H?-CB, H?-MCH, H?-SO, HJ-CL, MA-PAN, M?-CH, MJ-T y Cr-MCR (macho infante hijo de HA-MCR).

El grupo del Zoológico El Pinar estaba constituido por 8 individuos: 5 hembras (3 adultas) y 3 machos (un adulto), identificados como: HA-CHU, HA-OL, HA-MN, HJ-T, HJ-LOQ, MA-P, MJ-CHL, MJ-PTO. La composición del grupo cambió al final del muestreo por la muerte de MA-P y HA-CHU y la introducción de individuos sustitutos que permanecieron en una jaula de aclimatación hasta el final del periodo de observación.

Observación y registro de las conductas: se utilizaron tres tipos de muestreo: focal, de barrido y ad libitum. El muestreo focal 
consistió en observar un individuo por un período de tiempo específico (Altman 1974, Lehner 1996), en este caso durante 15 min continuos. El muestreo de barrido consistió en observar sucesivamente (uno tras otro) todos los individuos del grupo, sólo el tiempo necesario para registrar su conducta. El muestreo ad libitum consistió en anotar lo que fuese llamativo en un determinado momento en cualquier individuo, y fue útil para el registro de comportamientos poco frecuentes (Altman 1974, Lehner 1996). Se realizó una observación focal por individuo cada día en cada parque, excepto en Caricuao porque no siempre fue posible localizar a todos los individuos, y un barrido por cada hora de observación focal.

Las observaciones se realizaron en dos periodos: desde mayo a noviembre de 2000 y desde marzo a abril de 2001, de lunes a viernes entre las 0900 y las $1700 \mathrm{~h}$. El orden de observación de los grupos fue diferente cada semana, así como el orden de observación diario de los individuos dentro cada grupo. En Caricuao, los individuos focales fueron observados según eran localizados mientras se recorría su área de vivienda. En los otros parques el orden de observación se estableció aleatoriamente cada día. Las observaciones se realizaron a corta distancia de los individuos en todos los parques, pero ocasionalmente se utilizaron binoculares en el zoológico Caricuao. Lo observado era registrado en un grabador de reportero y las estimaciones de la duración de los eventos se realizaron utilizando un cronómetro.

A partir de estas observaciones se determinó el repertorio de conductas sociales en cada grupo. Además, para cada conducta, se estimó la duración de cada evento (en segundos), el tiempo (en segundos) dedicado por individuo por periodo focal y la frecuencia por individuo por día. Las relaciones de rango y de afiliación se estimaron tomando en cuenta el número de interacciones agonísticas o afiliativas en las que participaba cada individuo como iniciador y como receptor. Se elaboraron matrices de agonismo y de afiliación siguiendo el procedimiento descrito por Lehner (1996). Cada individuo en el grupo fue listado a lo largo la primera fila y la primera columna de la matriz. La primera columna correspondió a los iniciadores y la primera fila a los receptores. En las restantes casillas se colocó el número de veces que un individuo inició una interacción con cada uno de los otros individuos. Luego se ordenó la matriz de manera que el individuo que más inició y menos recibió interacciones agonísticas quedó en el extremo superior izquierdo de la matriz y el menos inició en el inferior. En el caso de la matriz de afiliación, se colocó en el tope de la matriz a aquel individuo que recibió el mayor número de interacciones, y en el último lugar el que recibió el menor número. La dominancia relativa se estimó utilizando un índice basado en el propuesto por Beilhraz y Cox (1967). Este índice considera la proporción de interacciones agonísticas iniciadas por cada individuo en una díada determinada y se calcula según: $\operatorname{IDR}_{i j}=\left(\mathrm{I}_{i} / \mathrm{N}_{i j}\right)-\left[\left(\mathrm{N}_{i j}-\mathrm{I}_{i}\right) /\right.$ $\mathrm{N}_{i j}$ ], donde: $\mathrm{I} i$ es el número de interacciones iniciadas por el individuo $i$ hacia $j, \mathrm{y} \mathrm{N}_{i j}$ es el número de interacciones entre $i$ y $j$. Este índice varia entre $-1 \mathrm{y}+1$.

Análisis de los resultados: se determinó si existían diferencias en la duración, el tiempo dedicado por individuo por periodo focal a cada conducta, y la frecuencia de cada conducta por individuo por periodo de observación focal entre grupos (parques). Se utilizó la prueba Kruskal-Wallis, ya que los datos no cumplieron con los supuestos de homogeneidad de varianzas y normalidad requeridos por los modelos paramétricos. Se utilizó U Mann-Whitney como prueba a posteriori. Los resultados se reportan como medias y desviaciones estándar con el fin de hacerlos comparables con lo publicado por otros autores.

Las matrices de agonismo fueron analizadas cualitativamente debido a que muchos pares de individuos nunca interactuaron. Las matrices de afiliación se analizaron cuantitativamente utilizando el análisis de residuales basado en la prueba de Chi-cuadrado (Aguilar 2000). Este análisis permite determinar, de manera rápida y sencilla, qué valores se desvían significativamente de lo esperado por azar, comparando el 
valor del residual con un valor crítico (en este caso, $Z_{\text {crit }}= \pm 2.58, \alpha=0.01$ ). Residuales positivos superiores a ese valor indican asociación entre las variables y viceversa (repulsión entre las variables). También se analizó la posible asociación entre el número de interacciones agonísticas y afiliativas iniciadas por cada individuo y entre el número de interacciones agonísticas recibidas y el número de interacciones afiliativas iniciadas utilizando el coeficiente de correlación de rangos de Spearman.

\section{RESULTADOS}

Se completaron 42 h 46 min de observación en Caricuao (104 periodos focales), 44 h $58 \mathrm{~min}$ en Parque del Este (109 periodos focales) y 33 h 43 min. El Pinar (99 periodos focales). El esfuerzo focal por individuo fue similar en cada parque (Cuadro 1).

Repertorio de conductas sociales: Los tres grupos mostraron las siguientes conductas sociales:

Interacciones afiliativas (incluye juego social): acicalar (uno o varios individuos revisan el pelaje de otro con las manos y/o los dientes), solicitar acicalamiento (acostarse sobre el dorso, el vientre o de lado, frente a otro individuo, mirándolo y vocalizando), inspeccionar la zona ano genital de otro individuo (hurgar con las manos, mirar y oler), inspeccionar los dientes (hurgar con las manos o mirando de cerca), seguir a otro individuo (caminar detrás de un individuo), contacto físico no agonístico (colocar la mano, la cabeza o cualquier otra parte del cuerpo sobre otro individuo), montarse sobre la espalda de otro individuo y desplazarse con él de esta manera, estar junto a otro individuo, sentado o acostado a una distancia menor a 50 $\mathrm{cm}$, jugar con otro individuo (mordisquear, perseguir, agarrar, halar la cola de otro individuo, brincar, colgarse de las ramas o cuerdas con otro individuo).

Interacción sexual: entre individuos del mismo sexo y de sexos diferentes, agarrar por
CUADRO 1

Esfuerzo muestral por individuo en cada parque

TABLE 1

Sampling effort for individual at each Zoo

\begin{tabular}{|c|c|}
\hline Grupo & Identidad de los individuos \\
\hline \multirow[t]{11}{*}{ Caricuao } & MA-MR \\
\hline & MA-MC ${ }^{(1)}$ \\
\hline & M?-BB \\
\hline & M?-FL \\
\hline & HA-MA \\
\hline & HA-NEG \\
\hline & HA-CB \\
\hline & MJ-TA \\
\hline & $\mathrm{MJ}-\mathrm{CO}$ \\
\hline & HJ-TI \\
\hline & $\mathrm{HJ}-\mathrm{CH}$ \\
\hline \multirow[t]{12}{*}{ Parque del Este } & MA-PAN \\
\hline & $\mathrm{M} ?-\mathrm{CH}$ \\
\hline & HA-NE \\
\hline & HA-MCR \\
\hline & HA-B \\
\hline & HA-PN \\
\hline & $\mathrm{H}$ ?-CB \\
\hline & H?-MCH \\
\hline & H?-SO \\
\hline & MJ-T \\
\hline & HJ-CL \\
\hline & Cr-MCR \\
\hline \multirow[t]{9}{*}{ El Pinar } & MA-P (2) \\
\hline & HA-CHU (3) \\
\hline & HA-OL \\
\hline & HA-MN \\
\hline & MJ-CHL \\
\hline & MJ-PTO \\
\hline & HJ-T \\
\hline & HJ-LOQ \\
\hline & TOTAL \\
\hline
\end{tabular}

$\mathrm{N}$ representa el número de observaciones focales por individuo.

(1) Retirado en marzo de 2001.

(2) Murió el 3 de marzo de 2001.

(3) Murió el 10 de marzo de 2001.

$\mathrm{N}$ represents the number of focal sessions for individual. 
la cintura, luego montar sobre la espalda del otro (o dejarse montar) y realizar movimientos de pelvis. Sólo se observó en los grupos de Caricuao y El Pinar.

Cuidado materno y alo materno: conductas típicamente maternas (amamantar, acicalar, oler, lamer a la cría, colocar la cría sobre la espalda, en reposo o desplazándose) expresadas por hembras hacia infantes que son sus crías (cuidado materno) o no (cuidado alo materno) o por otros individuos, excepto machos, hacia la cría recién nacida o de pocas semanas (no incluye amamantar).

Interacciones agonísticas: mostrar los dientes, empujar, perseguir, halar el pelaje o la cola, morder, suplantar (desplazar a otro individuo de la posición que ocupa), sumisión (después de una interacción, agacharse dirigiendo la mirada al otro individuo, sólo observada en Parque del Este y El Pinar).

Diferencias cuantitativas entre grupos: se encontraron diferencias significativas entre grupos en la duración y en la frecuencia de las interacciones afiliativas (Kruskal-Wallis: Duración, $\mathrm{H}_{(2,31)}=13.10$, $\mathrm{p}=0.0014$, Frecuencia, $\left.\mathrm{H}_{(2,31)}=6.47, \mathrm{p}=0.039\right)$. Los análisis a posteriori indicaron que las conductas afiliativas fueron más largas en Caricuao que en los otros grupos (Fig. 1, U Mann Whitney: Caricuao-Parque del Este, $Z=-2.71, p=0.007$; Caricuao-Pinar,
$\mathrm{Z}=3.06, \mathrm{p}=0.002$ ), pero menos frecuentes en el grupo de Caricuao que en los grupos del Parque del Este y El Pinar (Fig. 2, U Mann Whitney: Caricuao-Parque del Este, $\mathrm{Z}=2.46, \mathrm{p}=0.014$, Caricuao-Pinar, $Z=-1.98, p=0.05$ ). También hubo diferencia entre grupos en la frecuencia de interacciones agonísticas (Kruskal-Wallis: $\left.\mathrm{H}_{(2,31)}=13.09, \mathrm{p}=0.0014\right)$. Los análisis a posteriori indicaron que las conductas agonísticas fueron más frecuentes en el Parque del Este y el Pinar que en Caricuao (Fig. 3, U Mann-Whitney: Caricuao-Parque del Este, $Z=3.55, p=0.0004$; Caricuao-Pinar: $\mathrm{Z}=2.48, \mathrm{p}=0.013$ ).

No hubo diferencia en el tiempo total dedicado a las conductas afiliativas o agonísticas entre grupos (Cuadro 2, Kruskal-Wallis: $\mathrm{p}>0.05)$. Sin embargo, la distribución del tiempo en conductas afiliativas y agonísticas específicas fue cualitativamente diferente. Entre las conductas afiliativas, el grupo de Caricuao dedicó la mitad del tiempo al acicalamiento de otros individuos (Cuadro 3), mientras que en el grupo del Parque del Este, llevar a otro individuo sobre la espalda y jugar ocuparon más tiempo que el acicalamiento. Esto quizá se deba a que el grupo del Parque del Este tenía una mayor cantidad de jóvenes en relación a adultos que los demás grupos. En el grupo El Pinar, estar junto a otro individuo fue la principal conducta afiliativa, seguida por jugar y acicalar. De modo que la afiliación fue menos cercana en este grupo que en los demás, porque generalmente

CUADRO 2

Tiempo total (en segundos) dedicado a cada tipo de conducta social por individuo corregido por el número de periodos de observación focal

TABLE 2

Total time (in seconds) dedicated to each social behavior per individual (corrected by the number of focal sessions)

Conducta

Interacciones afiliativas

Interacciones agonísticas

Interacción sexual

Cuidado materno/alomaterno

Se indica el promedio \pm DE.

Figures represent mean $\pm \mathrm{SD}$.
Caricuao

$137.30 \pm 118.91$

$6.67 \pm 9.40$

$16.76 \pm 16.78$
Parque del Este

$180.89 \pm 90.8$

$9.69 \pm 9.38$

El Pinar

$110.84 \pm 84.64$

$11.72 \pm 10.65$

$0.72 \pm 1.34$

$2.68 \pm 7.97$ 


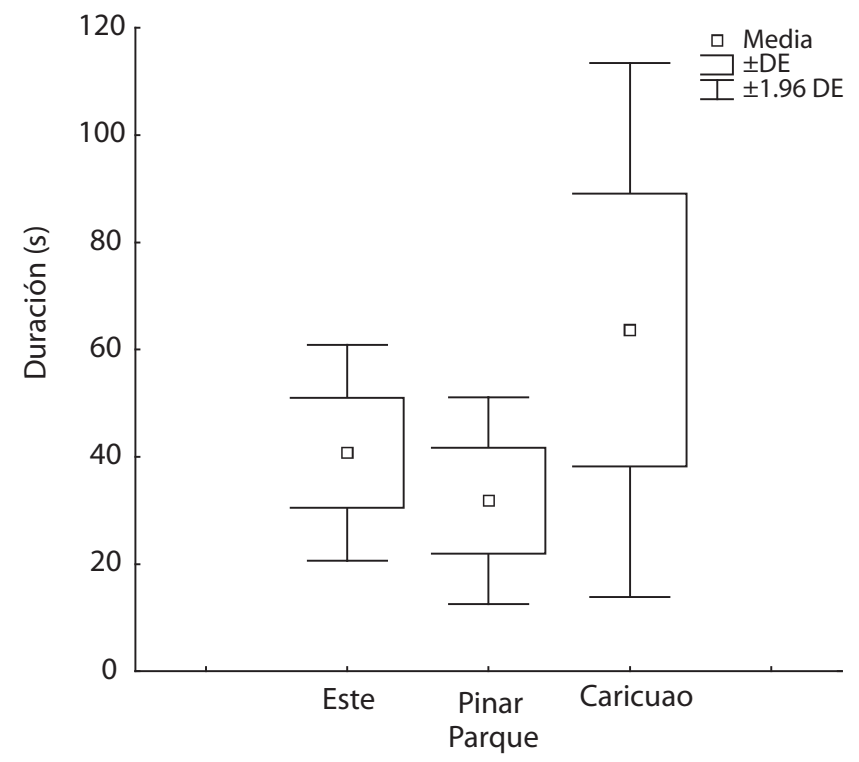

Fig. 1. Duración de las interacciones afiliativas en los tres grupos de estudio. Las diferencias entre el grupo de Caricuao y los demás grupos son significativas (U Mann-Whitney).

Fig. 1. Duration of affiliative interactions in the three study groups. Differences between Caricuao's group and the other two groups are significant (U Mann Whitney-Test).

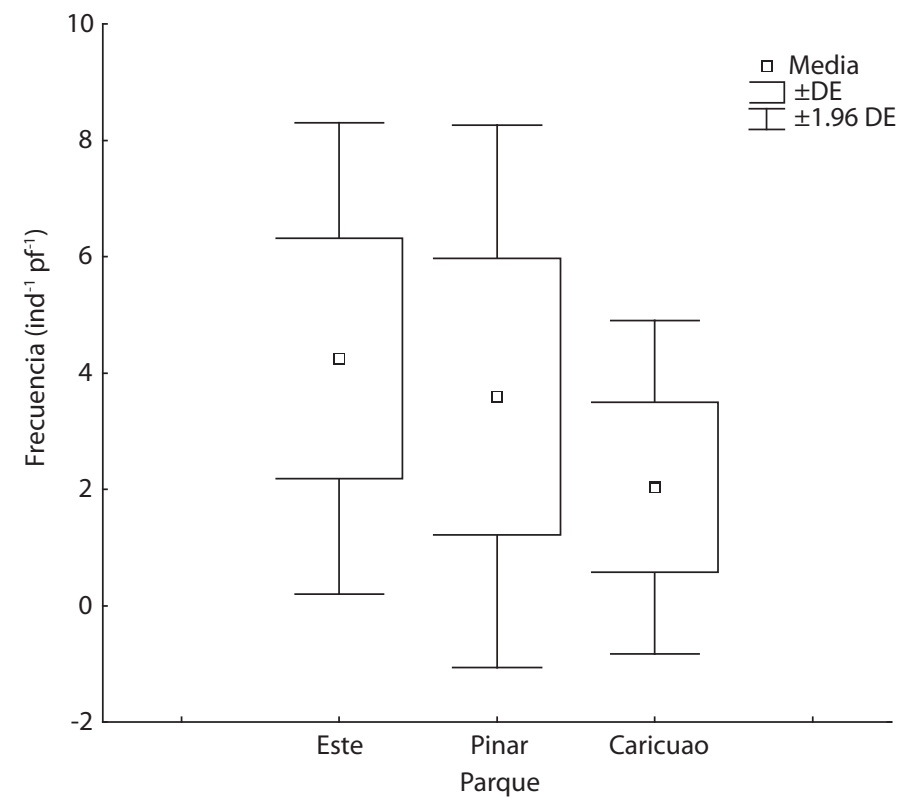

Fig. 2. Frecuencia de las interacciones afiliativas (por individuo por periodo focal) en los tres grupos de estudio. Las diferencias entre el grupo de Caricuao y los demás grupos son significativas (U Mann-Whitney).

Fig. 2. Frequency of affilaitive interactions (per individual and focal session) in the three study groups. Defferences between Cariacuao's group and the other two groups are significant (U Mann Whitney-Test). 


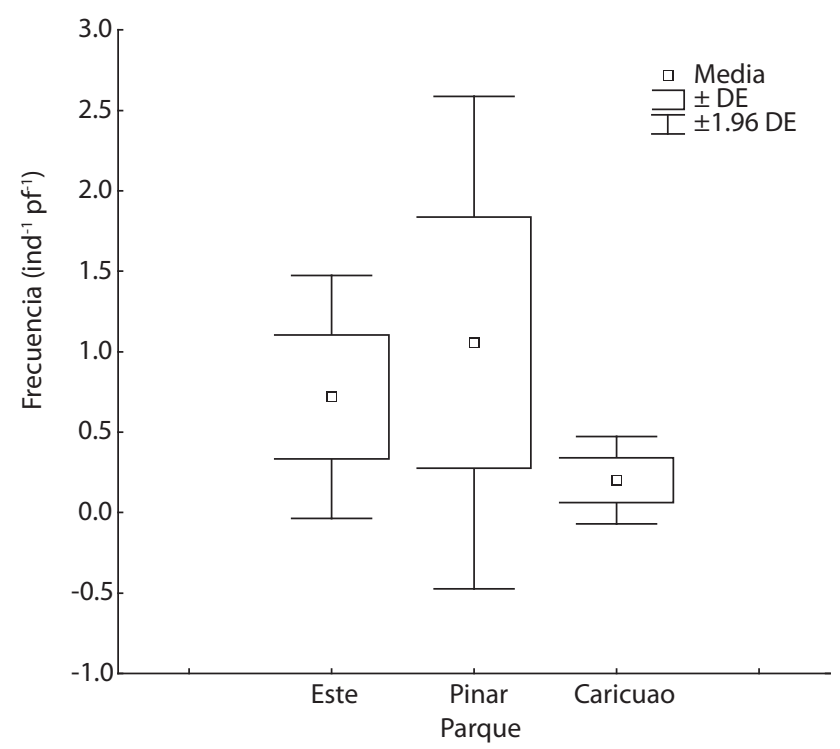

Fig. 3. Frecuencia de las interacciones agonísticas (por individuo por periodo focal) en los tres grupos de estudio. Las diferencias entre el grupo de Caricuao y los demás grupos son significativas (U Mann-Whitney).

Fig. 3. Frequency of agonistic interactions (per individual and focal session) in three study groups. Defferences between Cariacuao's group and the other two groups are significant (U Mann Whitney-Test).

no involucró contacto físico. Entre las conductas agonísticas, la agresión directa fue la más importante en los tres grupos.

Interacciones agonísticas: globalmente, la proporción de interacciones agonísticas iniciadas por hembras o machos varió entre grupos. Las hembras iniciaron más interacciones en el Parque del Este y en Caricuao; en El Pinar ambos sexos iniciaron interacciones en proporción similar (Cuadro 4). Los machos jóvenes iniciaron una proporción considerable de las interacciones agonísticas en Parque del Este y El Pinar.

Grupo Caricuao: se registraron 19 interacciones agonísticas: diez interacciones mientras MA-MC estaba en el grupo (cuatro entre hembras, cuatro entre machos y hembras y dos entre machos), y nueve en los tres días siguientes a su extracción (cinco entre hembras y machos, y cuatro entre machos). En el primer lapso, el individuo que más interacciones inició fue la hembra adulta HA-CB y los dirigió a juveniles (MJ-TA y HJ-CH) y a un macho adulto (MAMC). MA-MR, MA-BB y HA-MA iniciaron cada uno una sola agresión hacia M?-FL. Los jóvenes MJ-CO y HJ-CH iniciaron cada uno una agresión hacia el otro. Según lo anterior, HA-CB tendría mayor rango que las hembras jóvenes e incluso que el macho adulto. Las interacciones agonísticas en el grupo después de haber sido removido MA-MC fueron relativamente frecuentes respecto al período previo: nueve interacciones en cuatro días respecto a diez interacciones en diez días. En este lapso M?-BB fue el individuo que más interacciones recibió, tres de las cuales provinieron de las hembras adultas. M?-FL inició por primera vez una interacción dirigida hacia M?-BB.

Los índices de dominancia relativa calculados a partir de las escasas interacciones registradas no aportaron información adicional sobre la dinámica social en el grupo: HA-CB domina a las hembras juveniles y a MA-MC, y M?-FL era dominado por MA-MR, M?-BB y HA-MA. 
CUADRO 3

Diversidad e importancia relativa de conductas afiliativas y agonísticas especificas en los tres grupos

TABLE 3

Diversity and relative importante of affiliative and agonistic behaviors in the three study groups

Conductas

Caricuao

$103.50(54.9 \%)$

Acicalar

Solicitar acicalamiento

Oler zona anogenital

Seguir

Contacto físico

Estar junto a otro

Acarreo sobre espalda

Jugar

Total

Agonísticas

Agredir (1)

Perseguir

Suplantar

Total

$$
\begin{gathered}
0.67(0.4 \%) \\
13.32(7.1 \%) \\
4.67(2.5 \%) \\
33.03(17.5 \%) \\
3.17(1.7 \%) \\
30.28(16.1 \%) \\
188.63
\end{gathered}
$$

$$
\begin{gathered}
11.12(95.6 \%) \\
0.18(1.6 \%) \\
0.33(2.8 \%)
\end{gathered}
$$$$
11.63
$$

Parque del Este

El Pinar

$20.62(13 \%)$

$45.58(18 \%)$

$0.67(0.3 \%)$

$0.68(0.3 \%)$

$2.48(1.6 \%)$

$3.03(1.1 \%)$

$7.33(2.9 \%)$

$1.93(1.2 \%)$

$15.65(9.9 \%)$

$8.48(3.4 \%)$

78.93 (49.8\%)

99.57 (39.4\%)

87.43 (34.6\%)

252.78

38.77 (24.5\%)

158.38

$17.03(86.8 \%)$

$6.82(74.5 \%)$

$1.30(6.6 \%)$

$1.78(19.4 \%)$

$1.28(6.5 \%)$

$0.55(6.0 \%)$

9.15

19.62

Se indica el tiempo total (en minutos) dedicado por el grupo a cada conducta durante el periodo de muestreo en términos absolutos y porcentuales (entre paréntesis). (1) Empujar, halar el pelaje, morder.

Figures represent the total time (in minutes) and percent time (in parenthesis) dedicated to each behavior by the whole group during the sampling period. (1) Push, pull the coat, bite.

\section{CUADRO 4}

Proporción (\%) y frecuencia absoluta (entre paréntesis) de interacciones afiliativas y agonisticas según el sexo y edad de los individuos

TABLE 4

Proportion (\%) and absoulte frequency (in parenthesis) of affiiative and agonistic behaviors by sex and age

Caricuao Parque del Este El Pinar

\section{Interacciones afiliativas}

Iniciadas por hembras

Iniciadas por hembras adultas

Acicalamientos por hembras

Acicalamientos por machos

Interacciones agonísticas

Iniciadas por hembras

Iniciadas por machos adultos
76.3 (106)

$83.2(88)$

$82.3(167)$

$64.4(56)$

65.1 (69)

30.3 (10)

$49.1(82)$

25 (9)

63 (12)

74.5 (35)

11 (2)

$10.6(5)$
42.2 (27)

$57.1(32)$

50 (28)

$58.1(18)$

$13.5(5)$ 
Grupo Parque del Este: se registraron 47 interacciones agonísticas, 26 fueron entre hembras $(55 \%)$ y el resto entre machos y hembras $(45 \%)$. No se registraron interacciones entre machos, probablemente debido su bajo número, de modo que la estimación del rango de los machos dependió de sus interacciones con las hembras. Cualitativamente, HA-B y MA-PAN eran los individuos de mayor rango. HA-B dirigió interacciones a hembras (H?-CB y H?-SO) y a los jóvenes de ambos sexos, pero nunca recibió interacciones de otras hembras, aunque M?-CH le dirigió dos interacciones. A pesar que H?-CB inició más interacciones que ningún otro individuo en el grupo, nunca las dirigió a HA-B, H?-SO y MA-PAN, pero recibió interacciones de dos individuos adultos (HA-B y MA-PAN) y de una hembra joven (HJ-CL). Por su parte, MA-PAN dirigió cuatro interacciones a hembras juveniles (HJ-CL y $\mathrm{H}$ ?-MCH) y dos a hembras adultas, pero no a HA-B. Los índices de dominancia relativa corroboran los rangos de HA-B y MA-PAN, y además indicaron que las hembras eran dominantes sobre los machos con los que interactuaban. Aunque M?-CH fue el único macho que dirigió interacciones a una hembra adulta (HA-B), ésta dominaba sobre él.

Grupo El Pinar: se observaron 64 interacciones agonísticas, 19 entre hembras $(30 \%)$, diez entre machos $(15 \%)$ y el resto entre machos y hembras (55\%). El grupo de este parque presentó el mayor número de interacciones de los estudiados y también la mayor proporción de interacciones entre machos y hembras: 35 de 64, respecto a 21 de 47, en el Parque del Este. Según los registros, las hembras HA-OL y HA-CHU tenían el mayor rango en el grupo, seguidas por dos machos MJ-CHL y MA-P. Cuando se consideraron por separado las interacciones entre machos, MA-P fue el macho de mayor rango, seguido de MJ-CHL. La situación jerárquica de HA-OL y HA-CHU en el grupo se resolvió al considerar solamente las interacciones entre hembras. Según estos registros, HA-OL dirigió 11 interacciones a las hembras más jóvenes del grupo, mientras que
HA-CHU inició sólo una interacción. Una alta proporción de las interacciones iniciadas por HA-CHU fueron dirigidas a MJ-CHL, en parte debido a intentos de retaliación de éste sobre MJ-PTO (hijo de HA-CHU).

Los índices de dominancia relativa entre pares de individuos corroboran el análisis anterior: aunque MJ-PTO inició muchas interacciones, incluso hacia machos y hembras adultas, era subordinado frente a los dos machos. Las hembras adultas no dirigieron agresiones hacia MJ-PTO. MJ-PTO dominaba a dos de las hembras jóvenes pero no a MJ-MN.

MA-P y HA-CHU murieron durante el periodo de estudio y fueron introducidas dos hembras sustitutas que permanecieron varios días en una jaula dentro de la fosa. Durante este período se registraron muchas interacciones, principalmente dirigidas a las hembras enjauladas (9/13) que no podían responder a las agresiones. HA-OL y MJ-CHL eran entonces los individuos de mayor edad del grupo y dirigieron algunas interacciones a antiguos miembros del grupo (HJ-LOQ, MJ-PTO y HJ-TI), mientras que los jóvenes sólo lo hicieron hacia las hembras enjauladas. MJ-PTO, anteriormente propenso a iniciar interacciones, no inició ninguna pero recibió dos de la hembra dominante (HA-OL). El cambio en su conducta puede asociarse a la ausencia de su madre (HA-CHU).

Interacciones afiliativas: globalmente, las hembras iniciaron más interacciones afiliativas que los machos en todos los grupos, y en las hembras, una alta proporción estuvo representada por acicalamientos (Cuadro 4).

Grupo Caricuao: se registraron 139 conductas afiliativas en el grupo de las cuales, $79(57 \%)$ ocurrieron entre hembras, cinco (3\%) entre machos y el resto entre hembras y machos. Los machos iniciaron 25 interacciones hacia las hembras y las hembras iniciaron 30 interacciones hacia los machos. HA-MA y su cría (Cr-MA, macho infante) recibieron la mayoría de las interacciones afiliativas dentro del grupo, seguidas por HA-CB. Tres hembras 
iniciaron la mayor parte de las interacciones en el grupo: HA-NEG, HA-CB y HA-MA. HA-NEG y HA-CB dirigieron la mayoría de sus conductas hacia HA-MA pero sólo HA-CB dio contactos afiliativos a dos machos adultos (MA-MC y MA-MR). M?-BB y M?-FL fueron los únicos machos que no recibieron contactos afiliativos de las hembras. Entre los machos adultos, MA-MC dirigió la mayoría de las conductas a HA-MA (9/12).

Cuando se consideraron las interacciones que ocurrieron entre hembras se observó que todas iniciaron contactos afiliativos hacia HA-MA y su cría. Todas las hembras adultas dirigieron al menos una conducta afiliativa hacia las otras hembras del grupo, pero las hembras juveniles se concentraron principalmente en HA-MA y su cría. Las interacciones afiliativas entre machos fueron escasas: MA-MR y M?-BB intercambiaron un contacto afiliativo y M?-FL inició dos contactos pero no recibió ninguno.

Se encontraron afiliaciones significativas en once díadas, entre hembras, entre hembra y macho o entre macho y hembra (Cuadro 5, $\left.\chi_{90,0.05}^{2}=285.9, \mathrm{p}<0.01\right)$. Llama la atención la escasa asociación entre HA-MA y su cría y la alta asociación entre un macho adulto agresivo (MA-MC) y la cría. No hubo asociaciones ni repulsiones significativas entre machos. El nacimiento de las crías de HA-CB y de HA-NEG se asoció a un cambio en la distribución de las conductas afiliativas. HA-NEG y su cría pasaron a ser los individuos que más interacciones recibieron, seguidas de HA-CB; sin embargo, no hubo asociaciones significativas entre individuos durante este período $\left(\chi^{2}\right.$ $132,0.05=59.99, \mathrm{p}=0.34$ ).

Las asociación entre el número de interacciones afiliativas y el número de interacciones

CUADRO 5

Residuales estandarizados para las interacciones afiliativas entre individuos en el grupo Caricuao antes del nacimiento de las crías de HA-NEG y HA-CB

TABLE 5

Standarized residuals of affiliative interactions between pairs of individuals of Cariacuao group before the birth of HA-NEG and HA-CR young

R E C I B E

\begin{tabular}{|c|c|c|c|c|c|c|c|c|}
\hline & Cr-MA & HA-MA & HA-CB & MJ-TA & MJ-CO & HJ-TI & HJ-CH & MA-MC \\
\hline HA-MA & -6.50 & & & & & 3.49 & & 3.64 \\
\hline HA-CB & 4.10 & & & & & & 2.70 & \\
\hline
\end{tabular}

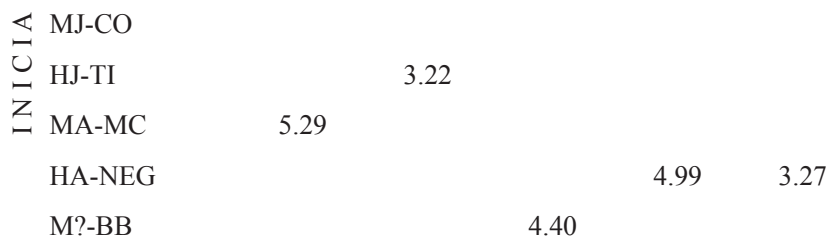

Sólo se muestran los residuales significativos.

Valores positivos indican más interacciones de las esperadas y viceversa.

La fila correspondiente a Cr-MA y la columna correspondiente a M?-FL no aparecen porque nunca inciaron o rebieron interacciones.

Los residuales no son simétricos; un individuo puede iniciar encuentros con otro sin que éste busque asociarse con él.

Only significant residuals are shown.

Positive values indicate more interactions than expected by chance and viceversa.

The row corresponding to Cr-MA and the column corresponding to M?-FL are not shown because they never initiated or received interactions.

Residuals are not symmetric; an individual can initiate interactions toward another without the latter initiating interactions toward it. 
agonísticas iniciadas por cada individuo no fue significativa (Spearman, $\mathrm{r} s=0.41, \mathrm{p}=0.06$ ). Tampoco se encontró relación entre el número de interacciones afiliativas iniciadas y el número de interacciones agonísticas recibidas (Spearman, $\mathrm{r} s=-0.40, \mathrm{p}>0.10)$. Cualitativamente, sin embargo, la hembra adulta de mayor rango (HA-CB) inició interacciones con más individuos que ningún otro miembro del grupo. HA-NEG también inició interacciones afiliativas con un gran número de individuos. Ambas hembras mostraron conductas afiliativas a muchos individuos pero las recibieron de menos.

Grupo Parque del Este: se registraron 203 interacciones de las cuales 151 (74\%) ocurrieron entre hembras, cinco (2\%) entre machos y el resto (24\%) entre machos y hembras. La cría (Cr-MCR) y su madre (HA-MCR) recibieron la mayoría de las interacciones, seguidas de HJ-CL. Las hembras iniciaron más interacciones y hacia más individuos en el grupo que los demás individuos. Los machos MJ-T y M?-CH fueron los que iniciaron menos interacciones. Cuando se consideraron las interacciones entre hembras por separado, se mantuvo el orden anteriormente descrito: la hembra dominante (HA-B) inició más interacciones pero la cría y su madre recibieron más que ella. En cuanto a las interacciones entre machos y hembras, MA-P fue el único macho que dirigió interacciones hacia la madre y la cría, y el que más interactuó con la hembra dominante. Las interacciones entre machos fueron escasas, pero destaca que M?-CH inició tres interacciones hacia otro macho (MJ-T). Se encontraron asociaciones y repulsiones significativas entre varios pares de individuos $\left(\chi_{110,0.05}^{2}=223.9, \mathrm{p}<0,01\right.$; Cuadro 6), generalmente entre hembras o entre hembras y machos. Como se esperaba, HA-MCR interactuó más de lo esperado con su cría, pero otra hembra (H?-SO) también se afilió a este infante. La única asociación significativa entre machos ocurrió entre M?-CH y MJ-T.

CUADRO 6

Residuales estandarizados para las interacciones afiliativas entre individuos en el grupo Parque del Este

TABLE 6

Standarized residuals of affiliative interactions between pairs of individuals of Parque del Este group

R E C I B E

Cr-MCR HA-MCR MJ-CL HA-B MJ-T HA-PN MA-PAN H?-CB HA-NE M?-CH

\begin{tabular}{|c|c|c|c|c|c|c|c|c|c|c|}
\hline HA-MCR & 4.21 & & & & & & & & & \\
\hline HA-B & & 3.13 & -2.63 & & & -3.01 & & 5.45 & 3.45 & \\
\hline HA-N & -3.86 & & & & 3.03 & & & & & \\
\hline $\mathrm{H}$ ?-CB & & & & 3.03 & & & & & & \\
\hline HA-NE & -4.62 & -4.02 & 3.64 & & & & 4.46 & & & 2.53 \\
\hline H?-MCH & & & & & & & & & & \\
\hline $\mathrm{H}$ ?-S0 & 7.38 & & & & & & & & & \\
\hline $\mathrm{M}$ ?-CH & & & & & 2.66 & & & & & \\
\hline
\end{tabular}

Sólo se muestran los residuales significativos.

Valores positivos indican más interacciones de las esperadas y viceversa.

La fila correspondiente a Cr-MCR no aparece porque nunca inició interacciones.

Los residuales no son simétricos; un individuo puede iniciar encuentros con otro sin que éste busque asociarse con aquél.

Only significant residuals are shown.

Positive values indicate more interactions than expected by chance and viceversa.

The row corresponding to Cr-MCR is not shown because he never initiated interactions.

Residuals are not symmetric; an individual can initiate interactions toward another without the latter initiating interactions toward it. 
En este grupo tampoco se encontró asociación entre el número de interacciones afiliativas y el número de interacciones agonísticas iniciadas por cada individuo (Spearman, $\mathrm{r}_{s}=0.41$, $\mathrm{p}=0.07$ ), ni entre el número de interacciones afiliativas iniciadas y el número de interacciones agonísticas recibidas (Spearman, $r s=-0.16$, $\mathrm{p}>0.10$ ).

Grupo El Pinar: se registraron 89 interacciones afiliativas, de las cuales 33 (37\%) ocurrieron entre hembras, diez (12\%) entre machos, y el resto entre machos y hembras. Este grupo presentó la menor proporción de interacciones hembra-hembra y la mayor proporción de interacciones macho-macho de los tres estudiados. HA-CHU inició más interacciones que los otros miembros del grupo, pero la mayoría fueron dirigidas a MJ-PTO (su hijo) y a HJ-LOQ. Si descontamos las interacciones que HA-CHU recibe de su hijo, HJ-LOQ fue la hembra que recibió más interacciones, seguida de HA-OL. Si se consideran solamente las interacciones entre hembras se encuentra que HA-CHU inició más interacciones y HA-OL recibió menos que las demás.

Se encontraron asociaciones significativas entre diez díadas $\left(\chi_{42,0.05}^{2}=126.5, \mathrm{p}<0.01\right.$, Cuadro 7), pero en ningún caso entre machos.
La matriz de relaciones afiliativas para el grupo mostró cambios después de la muerte de HA-CHU y MA-P. Entonces predominaron las interacciones entre individuos más jóvenes y subordinados, de modo que HJ-T inició, y HA-MN recibió, más interacciones que las demás hembras. HJ-T dirigió casi todas las interacciones a MJ-CHL, y HA-MN recibió todas las interacciones del joven MJ-PTO, siendo esta asociación la única significativa $\left(\chi_{16,0.05}^{2}=31.4, p=0.002\right.$, residual $\left.=2.77\right)$.

En este grupo tampoco se encontró relación entre el número de interacciones afiliativas y agonísticas iniciadas por cada individuo (Spearman, $r s=0.07, p>0.10$ ), ni entre el número de interacciones afiliativas iniciadas y el número de interacciones agonísticas recibidas (Spearman, $\mathrm{r} s=-0.43, \mathrm{p}>0.10$ ).

\section{DISCUSIÓN}

Los grupos de C. olivaceus observados mostraron repertorios de conductas sociales casi idénticos, pero la duración y la frecuencia de las conductas afiliativas y agonísticas difirieron entre ellos. Se discuten estas diferencias en detalle debido a que la estructura social de los grupos depende de las relaciones agonísticas y afiliativas. Se argumenta que las diferencias

CUADRO 7

Residuales estandarizados para las interacciones afiliativas entre individuos en el grupo El Pinar

TABLE 7

Standarized residuals of affiliative interactions between pairs of individuals of El Pinar group

R E C I B E

\begin{tabular}{|c|c|c|c|c|c|}
\hline & HJ-LOQ & MJ-PTO & HA-CHU & MA-P & HA-OL \\
\hline HJ-LOQ & & & & 3.21 & \\
\hline MJ-PTO & -3.52 & & 7.90 & & -2.69 \\
\hline HA-CHU & 3.31 & 5.59 & & -3.59 & -2.59 \\
\hline MJ-CHL & & & & & \\
\hline HJ-T & 3.66 & & & & 4.21 \\
\hline
\end{tabular}

Sólo se muestran los residuales significativos. Valores positivos indican más interacciones de las esperadas y viceversa. Los residuales no son simétricos.

Only significant residuals are shown. Positive values indicate more interactions than expected by chance and viceversa. Residuals are not symmetric. 
entre los grupos estudiados, o entre ellos y los naturales, pueden deberse a la condición del cautiverio. En todos los grupos se encontraron asociaciones (afiliativas y de rango) entre pares de individuos, sin embargo, la estructura de rangos dentro de los grupos no fue clara. Esto se discute en función del efecto del cautiverio sobre el valor inmediato del grupo.

\section{Relación entre cautiverio y agonismo:} en el presente estudio se encontró variación en el agonismo con la calidad del cautiverio, y diferencias cualitativas con lo descrito en condiciones naturales. Específicamente, la tasa de encuentros agonísticos fue mayor al desmejorar la calidad del cautiverio (mayor densidad de individuos, menor diversidad espacial del área de encierro), pero menor que en condiciones naturales. El confinamiento incrementa la probabilidad de encuentro entre individuos, la competencia por recursos, especialmente por el espacio individual, y además, puede generar stress propiciando las agresiones (Nieuwenhuijsen y de Waal 1982). Esto explicaría la mayor tasa de encuentros agonísticos en el grupo de El Pinar. Descartamos la posibilidad de que las diferencias entre grupos se deban a otros factores, como su composición sexual o etárea, porque la proporción de adultos (independientemente del sexo) a juveniles, fue similar entre parques. Aunque la proporción de sexos entre los adultos difería, esto no explica los resultados. Los grupos confinados tenían un solo macho adulto y tres o cuatro hembras adultas, de modo que la competencia (agresiones) por acceso a ellas, fue probablemente nula y no se corresponde con la alta frecuencia de interacciones en estos grupos. De modo que las diferencias en la calidad del confinamiento explicarían más fácilmente los resultados.

Por otra parte, aunque la información disponible para $C$. olivaceus en condiciones naturales es escasa, parece haber una diferencia notable con lo observado en cautiverio. Robinson (1981) registró 1067 interacciones agonísticas durante 70 días de observación (0.96 día ind $\left.^{-1}\right)$ en un grupo formado por 20 individuos (un macho adulto, dos subadultos, seis a ocho hembras adultas y cuatro infantes y seis juveniles). Cualitativamente, la tasa de encuentros agonísticos en el grupo estudiado por Robinson es superior a la encontrada en los grupos de El Pinar y Parque del Este: 64 y 47 interacciones en aproximadamente quince días (aprox. $0.53 \mathrm{i} \mathrm{día}^{-1}$ ind $^{-1}$ en El Pinar y $0.37 \mathrm{día}^{-1}$ ind $^{-1}$ en Parque del Este). Proponemos que la disponibilidad de alimento (abundante, estable y predecible), y la imposibilidad de mantener distancias interindividuales óptimas o de migrar del grupo, han podido favorecer una reducción de las interacciones en cautiverio. En relación a esto último, se ha propuesto que la convivencia prolongada, típica del cautiverio, e incluso el confinamiento excesivo, podrían conducir a la tolerancia o la evitación entre los individuos, reduciendo el agonismo a largo plazo (Nieuwenhuijsen y de Waal 1982, de Waal 2000). Entre los mecanismos de evitación más frecuentes está rehuir el contacto visual, mirando un punto fijo o mirando en todas direcciones (MacGuire et al. 1983, Cheney y Seyfarth 1989). Estas conductas fueron observadas en los dos grupos confinados (El Pinar y Parque del Este), pero no en el grupo que podía moverse libremente (López 2002), lo cual apoya nuestra conclusión.

\section{Relación entre cautiverio y afiliación:} en este estudio se encontró relación entre la condición de cautiverio y la frecuencia, y la duración de las interacciones afiliativas. Tradicionalmente se había propuesto que las conductas afiliativas, en primates, tenían como principales funciones mantener la cohesión del grupo y establecer coaliciones (McKenna 1978, Dunbar 1991). En las últimas décadas, se ha propuesto también que reducirían la tensión social (Schino et al. 1988), y mejorarían las relaciones dañadas después de las agresiones. Por tanto, las interacciones afiliativas promoverían la reconciliación (de Waal 1989, Aureli y de Waal 2000, Silk 2002). De ser esto cierto, habría más acercamientos y contactos en grupos socialmente tensos que en grupos estables. En concordancia con lo anterior, en el presente estudio encontramos más conductas afiliativas 
en los grupos confinados y con más agonismo (El Pinar y Parque del Este). Además, en el grupo de El Pinar, la afiliación estaba representada principalmente por acercamientos prolongados sin contacto físico. En una situación de roce social el contacto físico puede representar una trasgresión de los límites individuales. Acercarse, sin transgredir esos límites, puede aliviar la tensión y restaurar los nexos deteriorados por las agresiones (de Waal 1989, 2000, Silk 2002). Proponemos que la relativamente alta frecuencia de las interacciones afiliativas, así como su menor duración, en los grupos de El Pinar y el Parque del Este, resultarían de la tensión social, y serían formas de aliviarla.

Dinámica social en cautiverio: en los tres grupos observados había cierta estructura social, manifestada por la existencia de relaciones de rango y la formación de asociaciones afiliativas significativas entre díadas. Sin embargo, algunos aspectos de la dinámica social estaban parcialmente alterados. No existía, o no pudo determinarse, una estructura jerárquica clara y a que la relación entre el sexo o el rango y la propensión al agonismo o a la afiliación difirió de lo observado en condiciones naturales. En relación al primer punto, en primates sociales, la cohesión del grupo depende de que exista una jerarquía claramente definida y estable (Richards 1974). La cohesión se relaciona, a su vez, con el éxito en la competencia con otros grupos o en la defensa ante peligros potenciales (ej., depredadores). Proponemos que en cautiverio, el beneficio inmediato de la grupalidad disminuye o desaparece, y con él, la importancia de mantener la cohesión del grupo. Esto se reflejaría a su vez, en un sistema de rangos menos definido o inestable. Un indicador de que la cohesión en estos grupos creados artificialmente puede ser baja es que, en el grupo semicautivo (Caricuao), algunos individuos se ausentaban durante varios días, independientemente de su sexo o edad, o se alejaban considerablemente del resto del grupo.

En cuanto a la relación rango, sexo y agonismo, en condiciones naturales se ha observado que: el macho alfa domina a todos los individuos del grupo, incluso a la hembra dominante (Freese y Oppenheimer 1981), la mayoría de las interacciones agonísticas ocurre entre individuos de rango alto, y los machos juveniles reciben una proporción muy alta de las agresiones de la hembra alfa (Robinson 1981). En nuestro caso, en cambio, la mayoría de las interacciones ocurrieron entre individuos de rango bajo, las hembras adultas dominaban o tenían igual rango que los machos adultos y las dominantes no agredían a los jóvenes. Proponemos, que en estos grupos, el agonismo podría estar más relacionado con la excitabilidad de los individuos o su experiencia previa, que con el mantenimiento de una estructura social estable y funcional. Esto explicaría su alta frecuencia entre los jóvenes. Proponemos también que existe cierta tolerancia hacia individuos juveniles, por parte de las hembras adultas, probablemente asociada a la imposibilidad de mantenerlos a distancia o expulsarlos del grupo. La disrupción de la dominancia machohembra en los grupos observados sugiere que en cautiverio, el valor de los machos dominantes para las hembras (como proveedores de recursos y sostén de la estabilidad y cohesión del grupo) es bajo. Esto promovería cierta emancipación por parte de las hembras.

En cuanto a relación entre rango, sexo y afiliación, en el presente estudio los individuos de mayor rango (adultos) iniciaron más conductas afiliativas que los de rangos inferiores, y las hembras participaron en más interacciones que los machos. La inclinación de las hembras a la afiliación ha sido observada también en grupos silvestres y en cautiverio del género $(C$. olivaceus: Robinson 1981, O'Brien 1993; C. apella: Welter et al. 1990, Linn et al. 1995, Di Bitetti 1997). Sin embargo, sorprende que en los grupos observados en este trabajo, el macho y la hembra dominantes interactuaran pocas veces, cuando ésta es la díada más frecuente en otras especies del género. Las afiliaciones entre pares o grupos de hembras, comunes en condiciones naturales, tampoco fueron frecuentes en los grupos observados. Nuevamente, proponemos que en cautiverio, con escasos recursos que monopolizar, y sin el riesgo de 
invasiones por machos agresivos de otros grupos, el valor de los machos dominantes para las hembras es bajo. Asimismo, las alianzas entre hembras también perderían parte de su valor. Sin embargo, debe tenerse en cuenta que en grupos naturales, las hembras del grupo suelen estar emparentadas, lo cual favorece la cooperación entre ellas. Los grupos observados en este estudio, en cambio, estaban formados por individuos de diversa procedencia, generalmente no emparentados o que no crecieron juntos. Este factor también podría explicar la escasa afiliación entre hembras.

En resumen, en los grupos observados existe cierta estructura social aunque las relaciones entre los sexos y rangos están parcialmente alteradas. El efecto de las condiciones de vida en la conducta de los individuos se evidencia en una disrupción del sistema de relaciones sociales (patrones de agonismo alterados, jerarquía poco definida, evasión mutua, afiliación frecuente pero poco íntima). El acercamiento frecuente entre individuos en los grupos más confinados debe interpretarse con cuidado, pues si bien pareciera sugerir cohesión social puede ser un artefacto del cautiverio (Sommer et al. 2002). Por tanto, en estos grupos, la diferencia entre cohesión, socialidad y tolerancia mutua sería difícil de establecer. Las relaciones entre las hembras son más evidentes que entre los machos pero, en general, parecen existir mecanismos de evasión social, apaciguamiento y tolerancia. Éstos podrían atribuirse a la potencial tensión socio-ambiental a que están sometidos los individuos (confinamiento, imposibilidad de migrar, imposición de los miembros del grupo). Aunque el cautiverio afecta la conducta de los monos capuchinos observados, las conductas anormales severas reportadas en otros primates (auto mutilación y auto agresión) nunca fueron observadas, y las menos severas (movimientos estereotipados) ocurrieron rara vez (López 2002). Tampoco fueron observadas conductas como estimulación auto erótica o ruego, catalogadas como "indeseables" en individuos de exhibición (Mallapur y Choudhury 2003).

Para finalizar, el estudio del comportamiento de primates en zoológicos es una manera de establecer su bienestar y de lograr una experiencia agradable para los visitantes (Hosey 2005). Sin embargo, hasta ahora, ha sido difícil interpretar los resultados en función de las condiciones de cautiverio. En nuestro caso, sugerimos evaluar el bienestar de los individuos examinando también los niveles de hormonas típicamente asociadas al stress (corticoesteroides y glucocorticoides) (ver Ulyan et al. 2006), la condición del sistema inmune (por su relación con el stress y la reproducción), la actividad sexual y el éxito reproductivo, salud general y longevidad.

\section{AGRADECIMIENTOS}

Agradecemos a I. Cañizales por proponer la realización de este trabajo y proporcionar apoyo logístico, a E. A. Herrera por sus sugerencias al proyecto y a J.L. Pérez-Emán por sus comentarios al manuscrito.

\section{RESUMEN}

El confinamiento es una situación extrema para los primates con áreas de vivienda extensas, y su efecto sobre la conducta puede ser considerable. Cebus olivaceus es el primate más común en los zoológicos de Venezuela pero su conducta en esta condición no ha sido documentada. Para estimar el efecto del confinamiento sobre el comportamiento social de $C$. olivaceus, observamos tres grupos en zoológicos de Caracas (Caricuao, Parque del Este, El Pinar), que diferían en las condiciones de cautiverio. El grupo en Caricuao, podía desplazarse libremente en un área no cercada, mientras que en Parque del Este y El Pinar estaban en fosas relativamente pequeñas. Se determinó el repertorio de conductas sociales y la frecuencia, duración y tiempo dedicado por individuo a cada comportamiento. Se realizaron observaciones focales de cada individuo, barridos del grupo y observaciones ad libitum. Se estableció la dominancia relativa y las asociaciones afiliativas entre individuos. El repertorio de conductas sociales fue similar entre grupos y respecto a lo observado en condiciones naturales. Sin embargo, las conductas afiliativas fueron menos frecuentes pero más largas en el grupo de Caricuao, mientras que las conductas agonísticas fueron más frecuentes en Parque del Este y El Pinar. Además, el agonismo fue menos frecuente en los grupos observados que en la naturaleza. Las diferencias entre grupos se explican por la condición del cautiverio. Sugerimos que con el confinamiento incrementa la tensión social y la probabilidad de encuentros agonísticos, en contraposición, la afiliación reduciría esta 
tensión. Por otra parte, la convivencia prolongada, la imposibilidad de mantener distancias individuales óptimas o de migrar del grupo promoverían la reducción del agonismo, a largo plazo, lo que explicaría las diferencias con los grupos naturales. En los tres grupos hubo cierta estructura social (relaciones de dominancia, afiliaciones y repulsiones entre individuos), pero algunos aspectos de la dinámica social están alterados. Los rangos de dominancia no fueron claros, el macho alfa no dominaba a la hembra de mayor rango, los dominantes no interactuaban afiliativamente más que otros individuos, y las hembras no se afiliaban preferentemente con otras hembras. Estas observaciones son contrarias a lo descrito en la naturaleza. Globalmente C. olivaceus tolera bien el cautiverio porque presenta un repertorio de conductas similar al observado en la naturaleza y no muestra conductas anormales (auto agresión, movimientos estereotipados); sin embargo la dinámica social es afectada.

Palabras clave: socialidad, dominancia, agonismo, afiliación, cautiverio, estructura social, primate tropical, mono capuchino común, Cebus olivaceus.

\section{REFERENCIAS}

Aguilar, V.H. 2000. Utilización de varias técnicas estadísticas multivariadas para el análisis de dieta y de hábitat. Trabajo Especial de Grado. Escuela de Biología, Univ. Central de Venezuela, Caracas, Venezuela.

Altman, J. 1974. Observational study of behavior: sampling methods. Behaviour 49: 227-265.

Aureli, F. \& F.B.M. de Waal. 2000. Natural conflict resolution. Univ. California, Berkeley, California, EEUU.

Beilhraz, R.G. \& D.F. Cox. 1967. Social dominance in Swine. Anim. Behav. 15: 117-122.

Cheney, D.L. \& R.M. Seyfarth. 1989. Redirected aggression and reconciliation among Vervet Monkeys Cercopithecus aethiops. Behaviour 110: 258-275.

Defler, T.R. 1979. On the ecology and behavior of Cebus albifrons in eastern Colombia. II. Behavior. Primates 20: 491-502.

Di Bitetti, M.S. 1997. Evidence for an important social role of allogrooming in a platyrrhine primate. Anim. Behav. 54: 199-211.

Dunbar, R.I.M. 1991. Functional significance of social grooming in primates. Folia Primatol. 57: 121-131.

Fedigan, 1. 1993. Sex differences and intersexual relations in adult White-faced Capuchins (Cebus capucinus). Int. J. Primatol. 14: 853-877.
Fragaszy, D.M., E. Visalberghi \& J.G. Robinson. 1990. Variability and adaptability in the genus Cebus. Folia Primatol. 54: 114-118.

Freese, C. \& J. Oppenheimer. 1981. The Capuchin Monkeys, genus Cebus, p. 331-390. In A.F. CoimbraFilho \& R. Mittermeier (eds.). Ecology and behavior of neotropical primates. Academia Brasileira de Ciencias, Rio de Janeiro, Brasil.

Goldblatt, A. 1993. Behavioural needs of captive marine mammals. Aquatic Mammals 19: 149-157.

Grier, J. \& T. Burk. 1992. Biology of animal behavior. Mosby Year Book, San Louis, Missouri, EE.UU.

Hosey, G.R. 2005. How does the zoo environment affect the behaviour of captive primates? Appl. Anim. Behav. Sci. 90: 107-129.

Izawa, K. 1980. Social behavior of the wild Black-capped Capuchin (Cebus apella). Primates 21: 443-467.

Kinzey, W.G. 1997. Cebus, p. 248-257. In W.G. Kinzey. \& A. de Gruyter (eds.). New world primates. Ecology, evolution and behavior. Aldine de Gruyter, Nueva York, EEUU.

Lehner, P.N. 1996. Handbook of ethological methods. Univ. Cambridge, Cambridge, Inglaterrra.

Levy, L.E. 1984. Estudio del comportamiento de infantes del mono capuchino Cebus nigrivittatus en cautiverio. Trabajo Especial de Grado, Univ. Central de Venezuela, Caracas, Venezuela.

Linn, G.S., D. Mase, D., Lafrancois, R.T. O’Keeffe \& K. Lifshitz. 1995. Social and menstrual phase influences on behavior of group-housed Cebus apella. Am. J. Primatol. 35: 41-57.

López, M.C.H. 2002. Estudio comparativo del comportamiento del mono capuchino Cebus nigrivittatus (=olivaceus) en tres zoológicos de Caracas. Trabajo Especial de Grado. Univ. Central de Venezuela, Caracas, Venezuela.

Mallapur, A. \& B.C. Choudhury. 2003. Behavioral abnormalities in captive nonhuman primates. J. Appl. Anim. Welfare Sci. 6: 275-284.

Mallapur, A., N. Waran \& A. Sinha. 2005. Factors influencing the behaviour and welfare of captive Lion-tailed Macaques in Indian zoos. Appl. Anim. Behav. Sci. 91: 337-353.

Manson, J.H. \& S. Perry. 2000. Correlates of self-directed behaviour in wild White-faced Capuchins. Ethology 106: 301-317. 
McKenna, J.J. 1978. Biosocial function of grooming behavior among the Common Langur monkey (Presbytis entellus). Am. J. Phys. Antropol. 48: 503-510.

Nieuwenhuijsen, K. \& F. de Waal. 1982. Effects of spatial crowding on social behavior in a Chimpanzee colony. Zoo Biology 1: 5-28.

O'Brien, T.G. 1993. Assymetries in grooming interaction between juvenile and adult female Wedge-capped Capuchin Monkeys. Anim. Behav. 46: 929-938.

Perry, S. 1996. Female-female social relationships in wild White-faced Capuchin Monkeys Cebus capucinus. Am. J. Primatol. 40: 167-182.

Richards, S.M. 1974. The concept of dominance and methods of assesment. Anim. Behav. 22: 914-930.

Robinson, J. 1981. Spatial structure in foraging groups of Wedge-capped Capuchin Monkeys Cebus nigrivittatus. Anim. Behav. 29: 1036-1056.

Rowe, N. 1996. The pictorial guide to the living primates. Pogonias, Nueva York, EEUU.

Schino, G., S. Scucchi, D. Maestripieri \& P.G. Turillazzi. 1988. Allogrooming as a tension-reduction mechanism: a behavioral approach. Am. J. Primatol. 16: $43-50$.

Silk, J.B. 2002. The form and function of reconciliation in primates. Ann. Rev. Anthropol. 31: 21-44.
Sommer, V., A. Denham \& K. Little. 2002. Postconflict behaviour of wild Indian Langur Monkeys: avoidance of opponents but rarely affinity. Anim. Behav. 63: 637-648.

Ulyan, M.J, A.E. Burrows, C.A. Buzzell, M.A. Raghanti, J.L. Marcinkiewicz \& K.A. Phillips. 2006. The effects of predictable and unpredictable feeding schedules on the behavior and physiology of captive Brown Capuchins (Cebus apella). Appl. Anim. Behav. Sci. 101: 154-160.

Visalberghi, E. \& E. Addessi. 2000. Seeing group members eating a familiar food enhances the acceptance of novel foods in capuchin monkeys. Anim. Behav. 60: 69-76.

de Waal, F. 1989. Peacemaking among primates. Univ. Harvard, Cambridge, Massachusetts, EEUU.

de Waal, F. 2000. Primates. A natural heritage of conflict resolution. Science 289: 586-590.

Welker, C., P. Becker, H. Höhmann \& C. Schäfer-Witt. 1987. Social relations in groups of the Black-capped Capuchin Cebus apella in captivity. Interactions of group-born infants during their first six months of life. Folia Primatol. 49: 33-47.

Welker, C., P. Becker, H. Höhmann \& C. Schäfer-Witt. 1990. Social relations in groups of the Black-capped Capuchin Cebus apella in captivity. Folia Primatol. 54: 16-33. 